\title{
The relationship of blood glucose with cardiovascular disease is mediated over time by traditional risk factors in type 1 diabetes: the DCCT/EDIC study
}

\author{
Ionut Bebu $^{1}$ • Barbara H. Braffett ${ }^{1}$ • Rodica Pop-Busui ${ }^{2}$ - Trevor J. Orchard ${ }^{3}$. \\ David M. Nathan ${ }^{4}$ - John M. Lachin ${ }^{1}$ t the DCCT/EDIC Research Group
}

Received: 2 March 2017 / Accepted: 7 June 2017 /Published online: 16 July 2017

(C) Springer-Verlag GmbH Germany 2017

\begin{abstract}
Aims/hypothesis Chronic hyperglycaemia, as measured by $\mathrm{HbA}_{1 \mathrm{c}}$ levels, is a major risk factor for atherosclerosis and cardiovascular disease (CVD) in type 1 diabetes. Our aim was to describe the degree to which the effect of $\mathrm{HbA}_{1 \mathrm{c}}$ on the risk of CVD is mediated by its effect on traditional risk factors over time, and how these mediation pathways change over time.

Methods The DCCT and its observational follow-up study, the Epidemiology of Diabetes Interventions and Complications (EDIC), followed 1441 participants for a mean of 27 years, with periodic measurement of $\mathrm{HbA}_{1 \mathrm{c}}$ and risk factors over time. We assessed the proportion of the $\mathrm{HbA}_{1 \mathrm{c}}$ effect on risk of CVD that was mediated through its effects on systolic BP (SBP), pulse rate, triacylglycerols and LDL-
\end{abstract}

The complete list of the DCCT/EDIC Research Group is provided in the electronic supplementary material (ESM).

Electronic supplementary material The online version of this article (doi:10.1007/s00125-017-4374-4) contains peer-reviewed but unedited supplementary material, which is available to authorised users.

Ionut Bebu

ibebu@bsc.gwu.edu

1 The Biostatistics Center, The George Washington University, 6110 Executive Boulevard, Rockville, MD 20852, USA

2 Department of Internal Medicine, Division of Metabolism, Endocrinology and Diabetes, University of Michigan, Ann Arbor, MI, USA

3 Graduate School of Public Health, University of Pittsburgh, Pittsburgh, PA, USA

4 Massachusetts General Hospital, Harvard Medical School, Boston, MA, USA cholesterol (LDLc) levels, and how the proportion mediated changed over time.

Results The association of $\mathrm{HbA}_{1 \mathrm{c}}$ with $\mathrm{CVD}$ outcomes was stable over time, while that of traditional risk factors (SBP, pulse rate, triacylglycerols and LDLc) increased. At 10 years of followup, the effect of $\mathrm{HbA}_{1 \mathrm{c}}$ on 10 year CVD risk was minimally mediated by SBP $(2.7 \%)$, increasing to $26 \%$ at 20 years. Likewise, from 10 year follow-up to 20 year follow-up, the proportion of $\mathrm{HbA}_{1 \mathrm{c}}$ effect mediated through pulse rate increased from $6.3 \%$ to $29.3 \%$, through triacylglycerols from $2.2 \%$ to $22.4 \%$, and through LDLc from $9.2 \%$ to $30.7 \%$.

Conclusions/interpretation As participants age, the predictive association of mean $\mathrm{HbA}_{1 \mathrm{c}}$ on subsequent CVD events is increasingly mediated by its effect on standard risk factors. Thus, management of traditional non-glycaemic CVD risk factors may have increasing benefits in an ageing type 1 diabetes population with longstanding hyperglycaemia.

Trial registration: ClinicalTrials.gov NCT00360893 and NCT00360815.

Keywords Area under the curve - Cardiovascular disease . Mediation proportion - Type 1 diabetes

\begin{tabular}{|c|c|}
\hline \multicolumn{2}{|c|}{ Abbreviations } \\
\hline CVD & Cardiovascular disease \\
\hline EDIC & $\begin{array}{l}\text { Epidemiology of Diabetes Interventions and } \\
\text { Complications }\end{array}$ \\
\hline LDLc & LDL-cholesterol \\
\hline MACE & Major atherosclerotic cardiovascular events \\
\hline NIDDK & $\begin{array}{l}\text { National Institute of Diabetes and Digestive and } \\
\text { Kidney Diseases }\end{array}$ \\
\hline SBP & Systolic BP \\
\hline
\end{tabular}




\section{Introduction}

Despite progress in treatment, the risk of cardiovascular disease (CVD) in type 1 diabetes remains higher compared with that in the age-matched population without diabetes $[1,2]$. Over a mean follow-up of 17 years, the DCCT and its observational follow-up, the Epidemiology of Diabetes Interventions and Complications (EDIC) study, demonstrated that an average of 6.5 years of intensive diabetes therapy, resulting in a mean $\mathrm{HbA}_{1 \mathrm{c}}$ of $\sim 7 \%$ $(53 \mathrm{mmol} / \mathrm{mol})$ in participants, reduced the risk of any CVD event by $42 \%$, and the risk of non-fatal myocardial infarction or stroke or death from CVD (major atherosclerotic cardiovascular events [MACE]) by $57 \%$ compared with conventional therapy, which resulted in a mean $\mathrm{HbA}_{1 \mathrm{c}}$ of $\sim 9 \%(75 \mathrm{mmol} / \mathrm{mol})$ [3]. The robustness of the protective effect of 6.5 years of intensive therapy was further confirmed by an additional 10 years of follow-up [4]. Over the entire 28 years of follow-up, intensive therapy reduced the incidence of any CVD event by $30 \%$ and of MACE by $32 \%$ compared with conventional therapy [4], despite similar glycaemic levels for the majority of the EDIC follow-up period.

In order to provide adequate power to detect effects reliably using Cox proportional hazard models, further investigation of risk factors for CVD was embargoed until 100 participants in the former DCCT conventional group had experienced a CVD event. This landmark was reached in 2013 and a formal risk factor analysis was carried out. In a multivariable Cox model that also adjusted for age, duration of diabetes, use of angiotensinconverting-enzyme (ACE) inhibitors and a family history of myocardial infarction, the time-weighted updated $\mathrm{DCCT} / \mathrm{EDIC}$ mean $\mathrm{HbA}_{1 \mathrm{c}}$ was a stronger predictor of future CVD events than the updated mean systolic BP (SBP), pulse rate, LDL-cholesterol (LDLc), and current triacylglycerols (triacyclglycerol value at time of testing), which also predicted events [5].

These analyses, however, did not allow for changing covariate effects over time, nor did they address whether any of the effect of $\mathrm{HbA}_{1 \mathrm{c}}$ on CVD risk was mediated by the effect of $\mathrm{HbA}_{1 \mathrm{c}}$ on other CVD risk factors and whether this mediated effect varied over time. In this context, mediation analyses are important as poor glycaemic control is associated with dyslipidaemia and higher pulse rate and $\mathrm{BP}[1,6]$, while more intensive glycaemic control is associated with the reversal of these effects [7]. Therefore, we have assessed the temporal changes in the effects of $\mathrm{HbA}_{1 \mathrm{c}}$ and traditional CVD risk factors on the risk of CVD, and investigated potential pathways by which changes in CVD risk factors over time could mediate the effects of $\mathrm{HbA}_{1 \mathrm{c}}$ on the risk of CVD during the 28 year follow-up of the DCCT/EDIC cohort.

\section{Methods}

The methods of the DCCT and EDIC study have been described in detail [8]. Briefly, a total of 1441 participants with type 1 diabetes were randomly assigned to receive either intensive therapy $(n=711)$ with the goal of lowering blood glucose levels as close to the 'non-diabetic' range as safely as possible, or to conventional therapy $(n=730)$ aimed at avoiding symptoms related to hyper- or hypoglycaemia without specific glucose targets. The DCCT ended in 1993, after an average of 6.5 years of follow-up, and all participants were offered intensive therapy and referred to their healthcare providers for subsequent diabetes care. In 1994, $98 \%$ of the surviving DCCT cohort enrolled in the EDIC follow-up observational study and, after an additional 20 years of follow-up, $94 \%$ of the cohort survivors are still actively participating. The DCCT and EDIC protocols were approved by the institutional review boards of all participating centres and all participants provided written informed consent.

Cardiovascular risk factors The analyses presented are based on data obtained at annual visits during both the DCCT and EDIC study, which included a detailed medical history, physical examination (e.g. BP and pulse rate) and collection of bio-specimens (e.g. blood samples). Recognised and putative CVD risk factors were evaluated by standardised methods $[8,9]$. $\mathrm{HbA}_{1 \mathrm{c}}$ was measured using high-performance liquid chromatography, quarterly during the DCCT and annually during EDIC. Fasting lipids (triacylglycerols, total cholesterol and HDL-cholesterol) were measured in the central laboratory annually during DCCT and every other year during EDIC, and LDLc was calculated using the Friedewald equation [10]. The updated weighted mean of a covariate (e.g. $\mathrm{HbA}_{1 \mathrm{c}}$ ) measured at different intervals in DCCT vs EDIC was computed using all values up to a particular visit, with weights proportional to the time intervals between measurements.

Cardiovascular outcomes CVD events were ascertained based on the medical history and electrocardiogram and relevant available medical records, and were adjudicated by a committee masked to DCCT treatment group and $\mathrm{HbA}_{1 \mathrm{c}}$ levels. The composite CVD outcome was defined as time to the first occurrence of CVD death, non-fatal myocardial infarction, non-fatal stroke, subclinical myocardial infarction on ECG, angina confirmed by ischaemic changes with exercise tolerance testing or by clinically significant obstruction on coronary angiography, revascularisation (with angioplasty or coronary artery bypass) or congestive heart failure (paroxysmal nocturnal dyspnea, orthopnea or marked limitation of physical activity caused by heart disease) [11]. All participants with an initial CVD event that occurred prior to 31 December 2013 were included in these analyses. 
Statistical analysis The availability of longitudinal measurements throughout the DCCT/EDIC study allows for the dynamic evaluation of the temporal changes in the covariate effects on the risk of CVD events over time. Aalen additive hazard models [12] assessed the effects of covariates at 10 years of follow-up on the risk of CVD during the subsequent 10 years of follow-up (years 10 to 20). Only CVD events within this 10 year follow-up period were included. Participants with CVD events that had occurred prior to the 10 year visit were excluded and participants who did not experience an event within the subsequent 10 years were administratively right censored after year 20 . Additional models then assessed the effects of covariates measured up to the 11 year visit on the risk of CVD during the subsequent 10 year interval (years 11-21). Likewise, models assessed effects of covariates measured up to year 12 on CVD outcomes during years 12 22 , then the effects of those measured up to year 13 during years 13-23, up to models that assessed the effects of covariates measured at year 20 on the risk of CVD during years 20 30 . For each participant, the 10 year period of risk was computed to the day from the beginning of the period, e.g. for the 11-21-year models, the period of risk for a given participant spanned the time from 11.00 years after the participant's date of randomisation to 21.00 years after. The models employed the covariate values up to the most recent visit prior to the start of the 10 year period.

Based on our recent CVD risk factor assessment [5] that identified the most significant risk factors from an extensive panel of previously established and putative risk factors ('full model', ESM Table 1), models employed the updated mean values of $\mathrm{HbA}_{1 \mathrm{c}}$, SBP, pulse rate, LDLc and the 'current' value of $\log _{e}$ (triacylglycerols) at the beginning of each 10 year interval as fixed covariates. For example, the 10-20 year models employed the updated mean or current covariate value at year 10, the 11-21 year models used covariate values at year 11, and so on. Similar to the Framingham [13] and American College of Cardiology (ACC)/American Heart Association (AHA) [14] scores, all analyses herein address covariate effects on the risk of CVD within a 10 year time horizon. All analyses adjusted for age and duration of diabetes, both being well-known risk factors for CVD.

The AUC of the receiver operating characteristic (ROC) curve describes the predictive ability of a variable or a model, allowing for censored data [15]. It represents the probability that a participant who experienced an event has a higher risk score than a participant who is event free; $\mathrm{AUC}=0.5$ represents a risk factor that is not associated with the outcome, while $\mathrm{AUC}=1$ corresponds to perfect prediction.

Models with covariate (SBP, LDLc, triacylglycerols or pulse rate), age and diabetes duration ('Model 1'), age and diabetes duration only ('Model 2'), $\mathrm{HbA}_{1 \mathrm{c}}$, age and diabetes duration ('Model 3') and the full model ('Model 4', ESM Table 1) were considered. For each model, the AUC was computed for each of the 10 year models (10-20 years, 1121 years, etc.) to describe the changes over time in the predictive effect of that covariate on the subsequent 10 year risk of CVD. More specifically, using the 10-20 year interval as an example, the model with covariates measured at year 10 was used to compute the AUC for CVD events in year 10 alone, then for events in years 10-11, then years 10-12, etc.; the AUC value at a particular time point (say, year 15) describes the ability of the model with covariates evaluated at year 10 to predict CVD events up to that time point (i.e. year 15). The AUC values are presented for descriptive purposes only and no formal statistical tests were performed to compare the AUC values across different models.

Mediation analyses [16] were then employed to investigate whether the predictive effect of $\mathrm{HbA}_{1 \mathrm{c}}$ on CVD risk in each 10 year model could be explained by another covariate. Using SBP as an example, the total effect of mean $\mathrm{HbA}_{1 \mathrm{c}}$ on CVD was decomposed as the sum of the direct effect of $\mathrm{HbA}_{1 \mathrm{c}}$ (effect of $\mathrm{HbA}_{1 \mathrm{c}}$ on CVD) and the indirect effect of $\mathrm{HbA}_{1 \mathrm{c}}$ (effect of $\mathrm{HbA}_{1 \mathrm{c}}$ on CVD mediated through its effects on SBP) (Fig. 1). The mediation proportion (also called proportion explained) is the ratio of the indirect effect to the total effect (proportion explained $=$ indirect effect/total effect). Similar to the AUC calculations, the mediation pathways were assessed in a dynamic fashion in successive 10 year models (10-20, 11-21, etc.).

Linear regression models were employed to assess the effect of mean $\mathrm{HbA}_{1 \mathrm{c}}$ on potential mediators (such as SBP), while Aalen additive hazard models were used for the timeto-event CVD outcome [12]. The direct and indirect effects are functions of the coefficients in the two models (see ESM Mediation Analysis). In this case, the Aalen model was chosen for use rather than the Cox proportional hazards model because the resulting effects (described above) have a direct interpretation in terms of the number of CVD events attributable to the corresponding pathways.

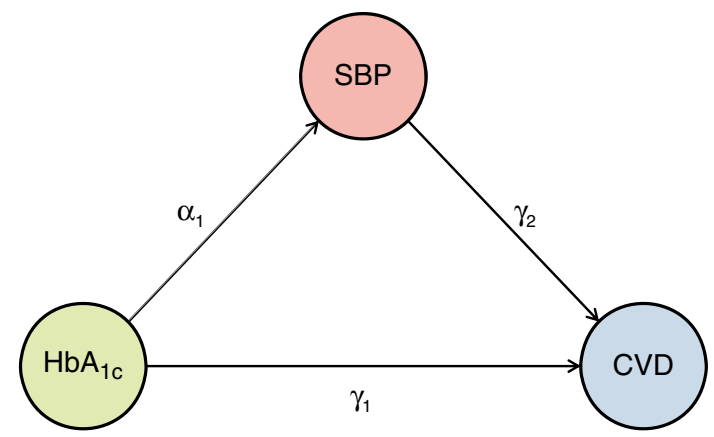

Fig. 1 The single mediator model, in which $\mathrm{HbA}_{1 \mathrm{c}}$ has a direct effect on CVD outcomes through the $\mathrm{HbA}_{1 \mathrm{c}}$ to CVD pathway, and an indirect effect on CVD is mediated by the effect of $\mathrm{HbA}_{1 \mathrm{c}}$ on SBP through the $\mathrm{HbA}_{1 \mathrm{c}}$ to SBP to CVD pathway. The total $\mathrm{HbA}_{1 \mathrm{c}}$ effect on CVD is the sum of the direct and indirect effects. The parameters $\alpha_{1}$ and $\gamma_{1} \gamma_{2}$ describe the effect of $\mathrm{HbA}_{1 \mathrm{c}}$ on SBP and the effects of $\mathrm{HbA}_{1 \mathrm{c}}$ and SBP on the risk of CVD, respectively (see ESM Mediation Analysis for details) 


\section{Results}

The number of participants at risk of CVD events in the DCCT/ EDIC cohort are presented for each 10 year interval in ESM Table 2. There were 184 participants who experienced at least one CVD event during DCCT/EDIC, with a median follow-up of 27 years since randomisation. The number of CVD events for each 10 year interval of the DCCT/EDIC are presented in ESM Table 2. The predictive value of each covariate was described using the AUC at each year, as depicted in Fig. 2. Fig. 2a-d shows the predictive value of covariate values (e.g. SBP) at year 10 on subsequent CVD risk during years 10-20, adjusted only for age and diabetes duration (Model 1; diamonds); the AUC values are cumulative over the 10-20 year period, e.g. the value at 15 years is the AUC for years 10-15 and the value at 20 years being the AUC for years 10-20. Fig. 2 also shows the AUCs for a model with age and diabetes duration alone (Model 2 ; circles), $\mathrm{HbA}_{1 \mathrm{c}}$, age and diabetes duration (Model 3; squares), and the AUCs for a model with all covariates adjusted for $\mathrm{HbA}_{1 \mathrm{c}}$, age and diabetes duration (Model 4; triangles). Adjusted for age and diabetes duration, mean $\mathrm{HbA}_{1 \mathrm{c}}$ (Model 3) performed similarly (similar AUC) to SBP (Model 1; Fig. 2a), but SBP was a stronger
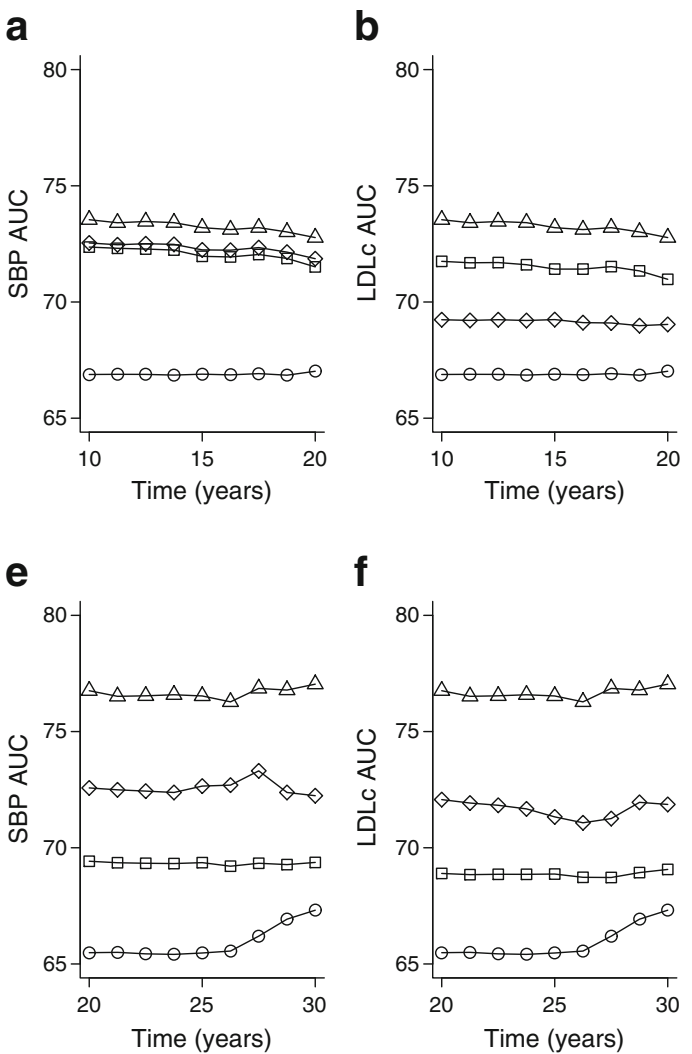

f

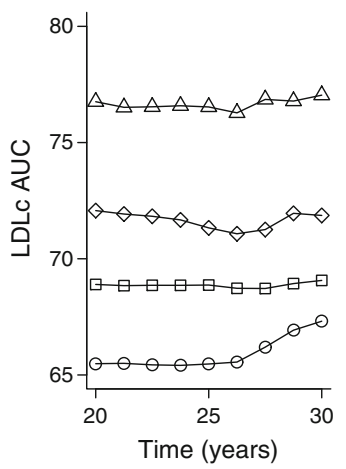

Fig. 2 AUC values for the updated mean SBP, LDLc levels, pulse rate or current triacylglycerol levels (Trigs), each adjusted for age and duration of diabetes (Model 1, diamonds) over (a-d) years 10-20 and (e-h) years 20-30 since enrolment. Also shown are the AUCs for models with age and diabetes duration alone (Model 2, circles), and age, diabetes duration predictor of CVD (larger AUC) than the other CVD risk factors (Fig. 2a-d; squares vs diamonds). In additional models using covariate values at year 15 to predict CVD events over the subsequent 10 years (i.e., 15-25 years), there were negligible differences between the AUC of $\mathrm{HbA}_{1 \mathrm{c}}$ and the other risk factors (data not shown).

Fig. 2e-h presents the AUC from models using the covariate values at year 20 to predict CVD events in years 20-30. Models with SBP, LDLc and triacyclglycerols (Model 1) had slightly higher AUCs than the model with $\mathrm{HbA}_{1 \mathrm{c}}$ (Model 3; Fig. 2e-g, diamonds vs squares), while the AUC for pulse rate was similar to that of $\mathrm{HbA}_{1 \mathrm{c}}$ (Fig. 2h, diamonds vs squares).

While the predictive ability of a model with only age and duration of diabetes (Model 2) remained approximately constant over time (Fig. 2, circles), adding mean $\mathrm{HbA}_{1 \mathrm{c}}$ to the model (i.e., Model 3) was more predictive (higher AUC) in the 10-20 year model than in the 2030-year model (Fig. 2, squares); the opposite was true for LDLc and triacylglycerols, but not for SBP and pulse rate (Fig. 2, diamonds). Moreover, the predictive ability of the full 20-30 year model (Model 4, described in ESM Table 1) was greater than that of the 10-20 year model (Fig. 2, triangles).
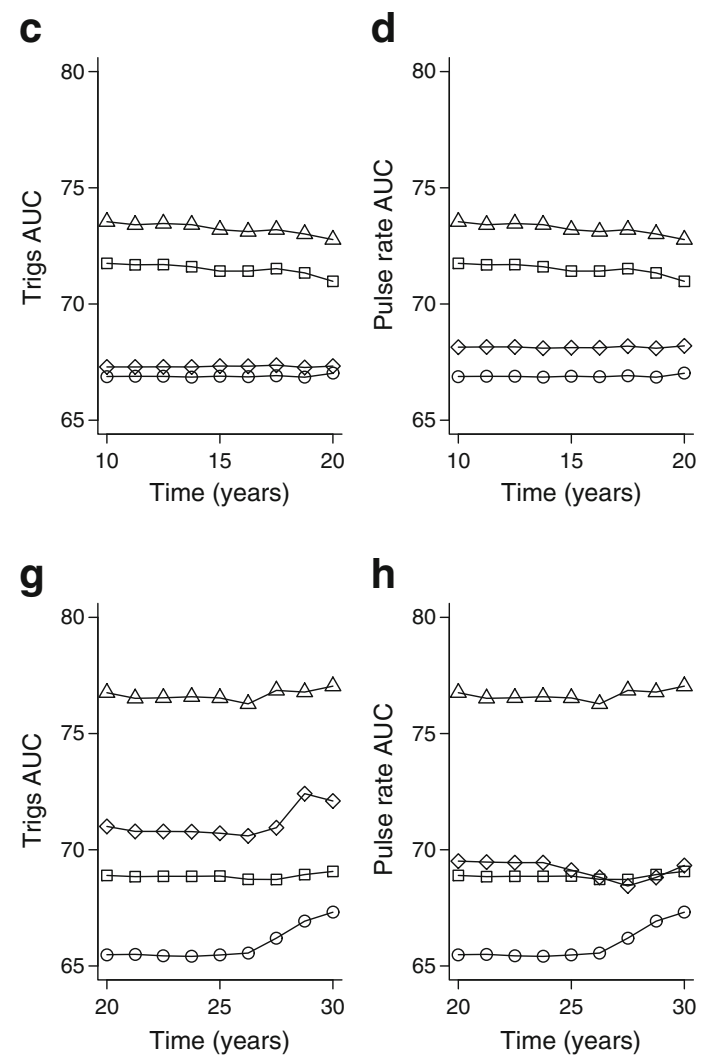

and $\mathrm{HbA}_{1 \mathrm{c}}$ (Model 3, squares), and the full model (Model 4, triangles). The data presented for the models with age and diabetes duration (Model 2, circles), age, diabetes duration and $\mathrm{HbA}_{1 \mathrm{c}}$ (Model 3, squares), and the full model (Model 4, triangles) are identical in (a-d) and in (e-h) 
Possible mediation pathways may explain the change in the AUC of mean $\mathrm{HbA}_{1 \mathrm{c}}$ over time. Among participants at risk at 10 years of follow-up, there were 89 participants with a CVD event in the subsequent 10-20 years, 97 with a CVD event between 15 and 25 years and 72 with a CVD event between 20 and 30 years. The total effect of mean $\mathrm{HbA}_{1 \mathrm{c}}$ on the risk of CVD outcomes in the successive 10 year models (10-20, 11-21, etc.) was approximately constant over time (ESM Fig. 1), with a small dip at year 16 . The average $\mathrm{HbA}_{1 \mathrm{c}}$ coefficient value in these Aalen models was approximately 0.0033 , indicating that for 1000 participants at risk, each 1 unit increase in mean $\mathrm{HbA}_{1 \mathrm{c}}$ (e.g. from $7 \%$ to $8 \%$, or a $10.9 \mathrm{mmol} / \mathrm{mol}$ increase) results in an average of $3.3(=0.0033 \times 1000)$ additional CVD events per year.

The decomposition of the total effect of the $\mathrm{HbA}_{1 \mathrm{c}}$ over time into the direct and indirect effects mediated by each of the other covariates is presented in Table 1 and demonstrates that the indirect effect of each covariate (mediation pathway) increases over time while the direct effect of $\mathrm{HbA}_{1 \mathrm{c}}$ decreases (Table 1 and ESM Fig. 1). For example, as noted above, in a model with $\mathrm{HbA}_{1 \mathrm{c}}$ as the exposure and SBP as the mediator, a $1 \%$ $\left(10.9 \mathrm{mmol} / \mathrm{mol}\right.$ ) higher $\mathrm{HbA}_{1 \mathrm{c}}$ in 1000 individuals at risk at year 10 resulted in a total expected number (total effect) of 3.3 additional individuals with CVD events per year over years 10-20; of these $3.21(97.3 \%)$ were attributable to the direct effect of $\mathrm{HbA}_{1 \mathrm{c}}$, and only $0.09(2.7 \%)$ to the indirect pathway through SBP. The $\mathrm{HbA}_{1 \mathrm{c}}$ values at year 15 had a higher indirect effect on the 15
25 year risk of CVD ( 0.51 events), and the values at year 20 had an even higher indirect effect on the 20-30 year risk $(0.88$ events). However, the total effect of the $\mathrm{HbA}_{1 \mathrm{c}}$ values at years 10,15 and 20 on risk of CVD in the subsequent 10 years remained approximately unchanged $(3.3,3.4,3.4$, respectively), and the direct effect declined modestly $(3.2,2.9,2.6$, respectively; Table 1).

Further, in mediation analyses, the SBP values at year 10 explained only $2.7 \%$ of the effect of the $\mathrm{HbA}_{1 \mathrm{c}}$ values at year 10 on the 10-20 year CVD risk, but the values at 15 years explained $15.0 \%$ of the $15-25$ year risk, and the values at 20 years explained $25.6 \%$ of the 20-30 year risk (Table 1 ).

Similar patterns were observed for the other three risk factors, with mediation proportion values over years 10-20 and $20-30$ of $6.3 \%$ and $29.3 \%$ for pulse rate, $2.2 \%$ and $22.4 \%$ for triacylglycerols and $9.2 \%$ and $30.7 \%$ for LDLc (Table 1). Figure 3 depicts the increasing fraction of the $\mathrm{HbA}_{1 \mathrm{c}}$ effect that is mediated by each of the other four factors over time, and ESM Table 3 presents a comparison of the mediation proportion values over time.

ESM Fig. 2 illustrates a model using both LDLc and SBP as mediators of the $\mathrm{HbA}_{1 \mathrm{c}}$ effect on CVD but assuming no association of LDLc with SBP. The resulting mediation proportions (Table 2 and ESM Fig. 3) range from $8.5 \%$ over years $10-20$ to $27.8 \%$ over years $20-30$ for LDLc, and from $2.5 \%$ to $23.8 \%$, respectively, for SBP.
Table 1 Participants with a CVD event during 10 year intervals, the total effect of a $1 \%(10.9 \mathrm{mmol} /$ mol) higher $\mathrm{HbA}_{1 \mathrm{c}}$ on CVD risk over time and its decomposition into the direct and indirect effects mediated through the updated mean of SBP, pulse rate, LDLc and triacylglycerol

\begin{tabular}{cccccc}
\hline $\begin{array}{l}\text { Variable/interval } \\
\text { (years) }\end{array}$ & $\begin{array}{l}\text { Participants with a CVD } \\
\text { event }(n)\end{array}$ & $\begin{array}{l}\text { Total } \\
\text { effect }\end{array}$ & $\begin{array}{l}\text { Direct } \\
\text { effect }\end{array}$ & $\begin{array}{l}\text { Indirect } \\
\text { effect }\end{array}$ & $\begin{array}{l}\text { Mediation proportion, \% } \\
(95 \% \text { CI })\end{array}$ \\
\hline SBP $^{\mathrm{a}}$ & & & & & \\
$10-20$ & 89 & 3.3021 & 3.2132 & 0.0888 & $2.69(0.46,6.43)$ \\
$15-25$ & 97 & 3.3927 & 2.8841 & 0.5085 & $14.99(8.27,27.16)$ \\
$20-30$ & 72 & 3.4499 & 2.5682 & 0.8817 & $25.56(13.41,53.82)$ \\
Pulse rate & & & & & \\
$10-20$ & 89 & 3.3223 & 3.1143 & 0.2080 & $6.26(-3.65,15.25)$ \\
$15-25$ & 97 & 3.3823 & 2.8464 & 0.5358 & $15.84(0.60,33.36)$ \\
$20-30$ & 72 & 3.4952 & 2.4714 & 1.0237 & $29.29(13.12,70.14)$ \\
Log $_{e}($ triacylglycerols) & & & & & \\
$10-20$ & 89 & 3.3158 & 3.2434 & 0.0724 & $2.18(-1.83,7.64)$ \\
$15-25$ & 97 & 3.4121 & 2.9116 & 0.5004 & $14.67(6.28,26.78)$ \\
$20-30$ & 72 & 3.4473 & 2.6745 & 0.7728 & $22.42(10.93,45.19)$ \\
LDLc $^{\mathrm{b}}$ & & & & & \\
$10-20$ & 89 & 3.3061 & 3.0013 & 0.3048 & $9.22(2.41,18.17)$ \\
$15-25$ & 97 & 3.3748 & 2.7132 & 0.6616 & $19.60(10.24,39.37)$ \\
$20-30$ & 72 & 3.4407 & 2.3828 & 1.0578 & $30.74(15.00,68.19)$ \\
\hline
\end{tabular}

All effects are per 1000 individuals at risk at the beginning of each interval

Using SBP and the 10-20 years interval as an example, a $1 \%(10.9 \mathrm{mmol} / \mathrm{mol})$ increase in $\mathrm{HbA}_{1 \mathrm{c}}$ is associated with approximately 3.3 additional CVD events per 1000 individuals per year at risk, of which $0.09(2.69 \%)$ CVD events are mediated through SBP

${ }^{\text {a }}$ Measured at the beginning of each interval

${ }^{\mathrm{b}}$ Value of $\log _{e}$ (triacylglycerols) at the beginning of each interval 
Fig. 3 (a-d) Proportion of the effect of $\mathrm{HbA}_{1 \mathrm{c}}$ mediated over time through the updated mean (a) SBP, (b) LDLc, (c) triacylglycerols and (d) pulse rate in 10 year models $(10-20,11-21$, etc.) using covariate values at the start of each interval
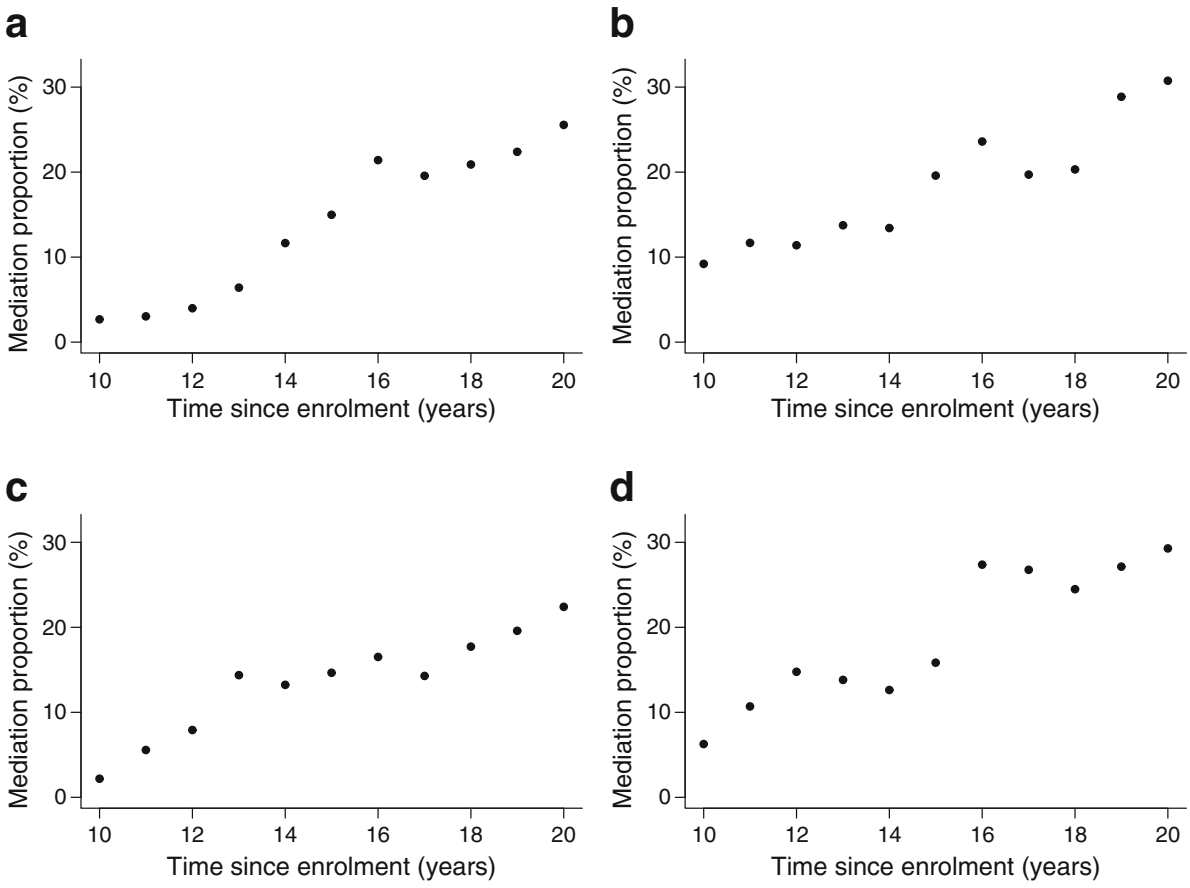

The results of a mediation analysis that include all four variables (SBP, pulse rate, triacylglycerols and LDLc) as mediators (ESM Fig. 4) demonstrate that, while $85.8 \%$ of the $\mathrm{HbA}_{1 \mathrm{c}}$ effect on CVD over years 10-20 was attributable to the direct $\mathrm{HbA}_{1 \mathrm{c}}$ path (14.2\% mediated by the other four covariates), this dropped to $58.3 \%$ over years $15-25$ (42.7\% mediation) and to $33.5 \%$ (66.5\% mediation) over years 20-30 (Table 3, Fig. 4). Over years 20-30, LDLc was the most important mediator of the $\mathrm{HbA}_{1 \mathrm{c}}$ effect (20.7\% proportion explained), followed by SBP $(18.7 \%$ proportion explained), pulse rate ( $15.2 \%$ proportion explained) and triacylglycerols (11.9\% proportion explained).

\section{Discussion}

These analyses demonstrate that the effect of mean $\mathrm{HbA}_{1 \mathrm{c}}$ on risk of CVD, when assessed in separate 10 year models starting at different points in time and adjusting for age and duration of diabetes, was largely constant over time. The coefficient was equivalent to an increase of approximately 3.3 CVD events per 1000 individuals per year for every $1 \%$ (10.9 mmol/mol) higher $\mathrm{HbA}_{1 \mathrm{c}}$. The 10 year predictive value (AUC) of $\mathrm{HbA}_{1 \mathrm{c}}$ declined slightly from about 0.72 over the 10-20 year interval to 0.69 over the 20-30 year interval, whereas the AUC of more traditional CVD risk factors (SBP, pulse rate, triacylglycerols and LDLc) increased slightly. The 10 year risk associated with each covariate remained largely unchanged over time. However, the proportion of the total $\mathrm{HbA}_{1 \mathrm{c}}$ effect on CVD risk that was mediated through its indirect effects on these CVD risk factors changed over time. Compared with the $14.2 \%$ of the $\mathrm{HbA}_{1 \mathrm{c}}$ effect on the 10 year CVD risk mediated by its indirect effects on SBP, pulse rate, triacylglycerols or LDLc over 10-20 years of follow-up, $66.5 \%$ of the $\mathrm{HbA}_{1 \mathrm{c}}$ effect on CVD risk was mediated by these other factors over 20-30 years of follow-up. Thus, while the total effect of $\mathrm{HbA}_{1 \mathrm{c}}$ remained unchanged over time, the
Table 2 Decomposition of the total effect of $\mathrm{HbA}_{1 \mathrm{c}}$ on $\mathrm{CVD}$ risk into the direct and indirect effects mediated by LDLc and SBP jointly

\begin{tabular}{|c|c|c|c|c|c|c|c|}
\hline \multirow[t]{2}{*}{ Interval (years) } & \multirow[t]{2}{*}{ Total effect } & \multirow[t]{2}{*}{ Direct effect } & \multicolumn{2}{|c|}{ Indirect effect } & \multicolumn{3}{|c|}{ Mediation proportion (\%) } \\
\hline & & & LDLc & SBP & Direct effect & LDLc & SBP \\
\hline $10-20$ & 3.298 & 2.937 & 0.279 & 0.082 & 89.1 & 8.5 & 2.5 \\
\hline $15-25$ & 3.413 & 2.367 & 0.576 & 0.469 & 69.4 & 16.9 & 13.7 \\
\hline $20-30$ & 3.423 & 1.657 & 0.952 & 0.814 & 48.4 & 27.8 & 23.8 \\
\hline
\end{tabular}

All effects are per 1000 individuals at risk at the beginning of each interval when the covariate values were measured

Using SBP and the 10-20 year interval as an example, a $1 \%(10.9 \mathrm{mmol} / \mathrm{mol})$ increase in $\mathrm{HbA}_{1 \mathrm{c}}$ is associated with approximately 3.298 additional CVD events per 1000 individuals per year at risk, of which $0.082(2.5 \%)$ CVD events are mediated through SBP 
Table 3 Decomposition of the total effect of $\mathrm{HbA}_{1 \mathrm{c}}$ on $\mathrm{CVD}$ risk into the direct and indirect effects mediated through SBP, pulse rate, triacylglycerols and LDLc in jointly adjusted models

\begin{tabular}{|c|c|c|c|c|c|c|c|c|c|c|c|}
\hline \multirow[t]{2}{*}{ Interval (years) } & \multirow[t]{2}{*}{ Total effect } & \multirow[t]{2}{*}{ Direct effect } & \multicolumn{4}{|c|}{ Indirect effect } & \multicolumn{5}{|c|}{ Mediation proportion (\%) } \\
\hline & & & SBP & Pulse & Trigs $^{\mathrm{a}}$ & LDLc & Direct effect & SBP & Pulse & Trig & LDLc \\
\hline $10-20$ & 3.304 & 2.835 & 0.082 & 0.139 & -0.027 & 0.274 & 85.8 & 2.5 & 4.2 & $-0.8^{\mathrm{b}}$ & 8.3 \\
\hline $15-25$ & 3.459 & 2.017 & 0.427 & 0.286 & 0.292 & 0.437 & 58.3 & 12.3 & 8.3 & 8.4 & 12.6 \\
\hline $20-30$ & 3.199 & 1.072 & 0.597 & 0.487 & 0.381 & 0.661 & 33.5 & 18.7 & 15.2 & 11.9 & 20.7 \\
\hline
\end{tabular}

Effects in jointly adjusted models over intervals of 10-20, 15-25 and 20-30 years using covariate values at 10, 15 and 20 years, respectively

Using SBP and the 10-20 year interval as an example, a $1 \%(10.9 \mathrm{mmol} / \mathrm{mol})$ increase in $\mathrm{HbA}_{1 \mathrm{c}}$ is associated with approximately 3.304 additional CVD events per 1000 individuals per year at risk, of which $0.082(2.5 \%)$ CVD events are mediated through SBP when factoring the effects of other variables

${ }^{\mathrm{a}}$ Trigs, $\log _{e}$ (triacylglycerols)

${ }^{\mathrm{b}}$ Mathematically, from the nature of the equation for the proportion explained (see ESM), it is possible that the proportion may be negative, which indicates that the proportion explained is virtually zero

patterns of mediation changed substantially as the cohort aged (Fig. 3, Table 3).

The change in the indirect effect of $\mathrm{HbA}_{1 \mathrm{c}}$ mediated through SBP, triacylglycerols, pulse rate and LDLc on CVD risk might be explained in several ways. The DCCT excluded individuals with high BP and hypercholesterolaemia at baseline. Thus, early in the study the only operant major risk factor was hyperglycaemia, which naturally predominated. It is also possible that hyperglycaemia has its major impact early in the course of CVD progression, e.g. in the early initiation of the atherosclerotic process. Conversely, SBP, triacylglycerols and LDLc may begin to affect CVD risk increasingly as individuals age, and the mean age of our cohort was only 27 at baseline. Indeed, the development of microalbuminuria and renal disease is known to worsen standard risk factors such as BP and lipids. In addition, there may be a cohort effect where participants with higher $\mathrm{HbA}_{1 \mathrm{c}}$ values experience CVD events earlier in the study and are no longer at risk later during follow-up, e.g. at 20 years.

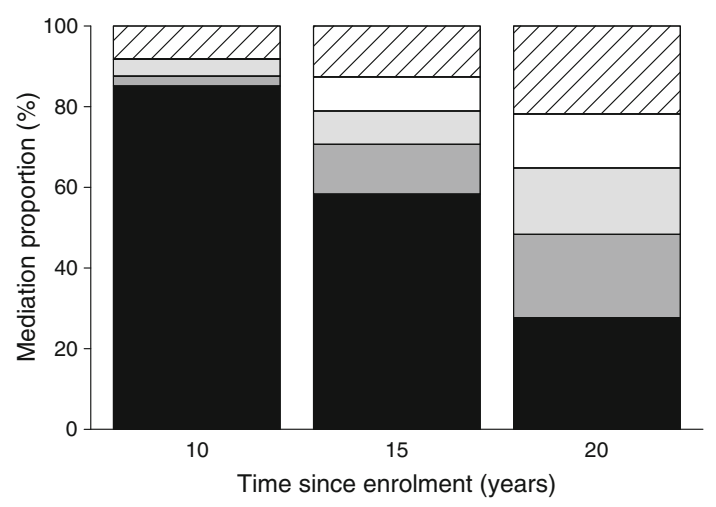

Fig. 4 Decomposition of the total effect of $\mathrm{HbA}_{1 \mathrm{c}}$ on CVD risk into the direct and indirect effects mediated through multiple risk factors over time for the 10-20, 15-25 and 20-30 year models, using covariate values at 10,15 and 20 years, respectively. Each shaded bar represents the proportion of the direct effect of $\mathrm{HbA}_{1 \mathrm{c}}$ on CVD risk (black) and the indirect effects through SBP (dark grey), pulse rate (light grey), triacylglycerols (white) and LDLc (diagonal stripes). Data are presented in Table 3
These mediation effects also apply to the net levels of SBP, LDLc and triacylglycerols as measured, regardless of whether or not the participant is receiving cardio-renal medical therapy (renin-angiotensin system [RAS] inhibitors, statins, etc.). It would be difficult to attempt to apply statistical modelling to estimate the mediation proportions in a population of individuals who were not receiving such medications. This is further complicated by the fact that medication use grew over time. At 10 years of follow-up, only $12.8 \%$ of participants were receiving a RAS inhibitor and $1.6 \%$ a statin/beta blocker. This usage increased to $58.3 \%$ and $51.8 \%$ at 20 years and $74.1 \%$ and $76.8 \%$ at 30 years, respectively. Further, using SBP as an example, anti-hypertensive medication use would translate into a lower indirect effect of $\mathrm{HbA}_{1 \mathrm{c}}$ on SBP and, subsequently, on CVD, and therefore a lower mediation proportion for the effect of SBP on CVD. As medication use increased, the mediation proportion for SBP is, if anything, an underestimate of the value without medication use.

These results have clinical implications and provide further insight into possible mechanisms leading to CVD in type 1 diabetes. Over 20-30 years of follow-up (27-37 years of diabetes duration), LDLc mediated $31 \%$ of the effect of $\mathrm{HbA}_{1 \mathrm{c}}$. Of the average additional 3.4 individuals with a CVD event per year associated with each $1 \%(10.9 \mathrm{mmol} / \mathrm{mol})$ increase in $\mathrm{HbA}_{1 \mathrm{c}}, \sim 1$ excess CVD event per year is therefore attributable to LDLc $(3.4 \times 0.31=1.05$ CVD events per year). This fraction of increased risk associated with $1 \%$ (10.9 mmol/mol) higher $\mathrm{HbA}_{1 \mathrm{c}}$ might be eliminated by treatments aimed to lower LDLc. Our findings further reinforce the value of aggressive glycaemic management early in the course of diabetes, followed by aggressive management of the other CVD risk factors as they become increasingly operant.

Regarding possible mechanisms, the year-to-year total effect (direct and indirect) of $\mathrm{HbA}_{1 \mathrm{c}}$ on the subsequent 10-year risk of CVD is relatively constant over time, suggesting aggressive glycaemic management has the potential to reduce the CVD risk throughout the course of diabetes. At the same time, the fraction 
of the $\mathrm{HbA}_{1 \mathrm{c}}$ effect on CVD risk that is mediated by its effects on these other covariates increases substantially over time. This finding suggests that aggressive management of traditional nonglycaemic CVD risk factors may further reduce the risk of CVD in those with longstanding diabetes and long-term glycaemic exposure above the target levels.

Acknowledgements A complete list of participants in the DCCT/EDIC Research Group is presented in the ESM published online for this article.

Data availability All DCCT/EDIC data files are available at the NIDDK Repository (www.niddkrepository.org/studies/edic).

Funding The DCCT/EDIC has been supported by cooperative agreement grants (1982-1993, 2012-2017) and contracts (1982-2012) with the Division of Diabetes Endocrinology and Metabolic Diseases of the National Institute of Diabetes and Digestive and Kidney Diseases (NIDDK) (current grant numbers U01 DK094176 and U01 DK094157), and through support by the National Eye Institute, the National Institute of Neurologic Disorders and Stroke, the General Clinical Research Centers Program (1993-2007) and Clinical Translational Science Center Program (2006-present), Bethesda, MD, USA. The following industry contributors had no role in the DCCT/EDIC study but have provided free or discounted supplies or equipment to support participants' adherence to the study: Abbott Diabetes Care (Alameda, CA, USA), Animas (Westchester, PA, USA), Bayer Diabetes Care (North America Headquarters, Tarrytown, NY, USA), Becton Dickinson (Franklin Lakes, NJ, USA), Eli Lilly (Indianapolis, IN, USA), Extend Nutrition (St Louis, MO, USA), Insulet Corporation (Bedford, MA, USA), Lifescan (Milpitas, CA, USA), Medtronic Diabetes (Minneapolis, MN, USA), Nipro Home Diagnostics (Ft Lauderdale, FL, USA), Nova Diabetes Care (Billerica, MA, USA), Omron (Shelton, CT, USA), Perrigo Diabetes Care (Allegan, MI, USA), Roche Diabetes Care (Indianapolis, IN, USA), and SanofiAventis (Bridgewater, NJ, USA). The study sponsor was not involved in the design of the study; the collection, analysis, and interpretation of data; writing the report; or the decision to submit the report for publication.

Duality of interest The authors declare that there is no duality of interest associated with this manuscript.

Contribution statement IB and JML wrote the initial draft of the manuscript. IB and BHB conducted statistical analyses. All authors contributed to the study design and the interpretation of the data, revised the manuscript for important intellectual content and approved the final version. IB is the guarantor of this work and, as such, had full access to all the data in the study and takes responsibility for the integrity of the data and the accuracy of the data analysis.

\section{References}

1. Orchard TJ, Costacou T, Kretowski A, Nesto RW (2006) Type 1 diabetes and coronary artery disease. Diabetes Care 29:2528-2538
2. Livingstone SJ, Looker HC, Hothersall EJ et al (2012) Risk of cardiovascular disease and total mortality in adults with type 1 diabetes: Scottish registry linkage study. PLoS Med 9: e1001321

3. Nathan DM, Cleary PA, Backlund JY et al (2005) Intensive diabetes treatment and cardiovascular disease in patients with type 1 diabetes. N Engl J Med 353:2643-2653

4. Diabetes Control and Complications Trial (DCCT)/Epidemiology of Diabetes Interventions and Complications (EDIC) Study Research Group (2016) Intensive diabetes treatment and cardiovascular outcomes in type 1 diabetes: the DCCT/EDIC study 30-year follow-up. Diabetes Care 39:686-693

5. Diabetes Control and Complications Trial/Epidemiology of Diabetes Interventions and Complications (DCCT/EDIC) Research Group (2016) Risk factors for cardiovascular disease in type 1 diabetes. Diabetes 65:1370-1379

6. Paterson AD, Rutledge BN, Cleary PA, Lachin JM, Crow RS (2007) Diabetes control and complications trial/epidemiology of diabetes interventions and complications research group: the effect of intensive diabetes treatment on resting heart rate in type 1 diabetes: the diabetes control and complications trial/epidemiology of diabetes interventions and complications study. Diabetes Care 30: 2107-2112

7. de Boer IH, Rue TC, Cleary PA et al (2011) Long-term renal outcomes of patients with type 1 diabetes mellitus and microalbuminuria: an analysis of the diabetes control and complications trial/epidemiology of diabetes interventions and complications cohort. Arch Intern Med 171:412-420

8. The DCCT/EDIC Research Group (1999) Epidemiology of diabetes interventions and complications (EDIC). Design, implementation, and preliminary results of a long-term follow-up of the diabetes control and complications trial cohort. Diabetes Care 22:99-111

9. The DCCT Research Group (1986) The diabetes control and complications trial (DCCT). Design and methodologic considerations for the feasibility phase. Diabetes 35:530-545

10. Friedewald WT, Levy RI, Fredrickson DS (1972) Estimation of the concentration of low-density lipoprotein cholesterol in plasma, without use of the preparative ultracentrifuge. Clin Chem 18:499-502

11. Lachin JM, Orchard TJ, Nathan DM (2014) DCCT/EDIC research group: update on cardiovascular outcomes at 30 years of the diabetes control and complications trial/epidemiology of diabetes interventions and complications study. Diabetes Care 37:39-43

12. Martinussen T, Scheike TH (2006) Dynamic regression models for survival data. Springer, New York

13. D'Agostino RBS, Vasan RS, Pencina MJ et al (2008) General cardiovascular risk profile for use in primary care: the framingham heart study. Circulation 117:743-753

14. Goff DC Jr, Lloyd-Jones DM, Bennett G et al (2014) American College of Cardiology/American Heart Association Task Force on Practice Guidelines: 2013 ACC/AHA guideline on the assessment of cardiovascular risk: a report of the American College of Cardiology/American heart association task force on practice guidelines. J Am Coll Cardiol 63:2935-2959

15. Heagerty PJ, Zheng Y (2005) Survival model predictive accuracy and ROC curves. Biometrics 61:92-105

16. Lange T, Hansen JV (2011) Direct and indirect effects in a survival context. Epidemiology 22:575-581 